\title{
Prognostic value of nucleolar morphometric variables in cytological breast cancer specimens
}

\author{
P J van Diest, J Mouriquand, N W Schipper, J P A Baak
}

\begin{abstract}
In a retrospective study on cytological specimens from 86 patients with histologically confirmed invasive breast cancer, the prognostic value of nucleolar morphometric variables was studied and compared with nuclear variables. One hundred nuclei and their nucleoli on each slide were measured with a graphic tablet system at a total magnification of $\mathbf{2 8 0 0}$ times using a stratified selection method. The number of nucleoli per 100 nuclei was also noted. Analysis of Kaplan-Meier univariate recurrence free survival curves showed significant differences for eight nuclear features, nine nucleolar features, and three combined nuclear and nucleolar variables. The total number of nucleoli per 100 nuclei was the best single prognostic variable. Multivariate survival analysis (Cox regression model) showed that no other feaures provided additional prognostic information beyond that given by the total number of nucleoli.

It is concluded that nucleolar morphometric variables assessed in cytological preparations have prognostic value in breast cancer, and the results of this study suggest that their prognostic value may exceed that of nuclear variables.
\end{abstract}

Since adjuvant chemotherapy strategies have become available for breast cancer, which improve survival on the one hand but have serious side effects on the other, only patients at high risk for relapse should be selected for this treatment. Although lymph node metastasis is widely used as a high risk indicator, ${ }^{1}$ it only predicts prognosis accurately in about $60-65 \%$ of all patients with breast cancer. ${ }^{2}$ Quantitative variables such as the mitotic activity index, the nuclear area, and the DNA index have been shown to enhance the accuracy of predictive prognosis to over $90 \%{ }^{2-4}$ Although this is a considerable and important improvement, there is still a need for alternative factors with additional prognostic value in breast cancer to increase the predictive accuracy of prognosis, ideally to $100 \%$. This is especially important as more small, lymph node negative tumours are being detected in screening programmes, in which lymph node state is therefore less important as a prognostic factor.

Subjective observations of prominent nucleoli in different types of cancer have aroused interest in the role of nucleoli in malignancy and hence prognosis. In recent publications, the value of nucleolar features has been studied in several tumours. ${ }^{5-7}$ In breast cancer there are also indications that the nucleolus is prognostically important,${ }^{8}$ and so far, one study has described the prognostic power of morphometric nucleolar variables in tissue sections. ${ }^{9}$

Tissue sections, however, are not very suitable for the study of nucleoli, as many nuclei are not present in their integrity because of sectioning, and eccentric nucleoli may therefore have been cut out. Furthermore, the widely used haematoxylin and eosin staining procedure is not ideal for the visualisation of nucleoli because of the relative hyperchromasia of nuclei. Contrary to this, cytological specimens, stained according to a hypochromic Papanicolaou procedure ${ }^{10}$ do not have these limitations, and are therefore especially suited to the quantitative analysis of nucleoli. Our aim, therefore, was to study the prognostic value of quantitative nucleolar features in cytological breast cancer specimens and compare these with nuclear variables.

\section{Methods}

Ninety eight patients diagnosed between 1975 and 1985 at the hospital attached to the University of Grenoble, France, were studied. The mean age was 54.5 (range $28-88$ ) years at the time of diagnosis, and the follow up was 56 months on average (range six-120). All patients were submitted to the same diagnostic procedure (physical examination, fine needle aspiration biopsy, mammography) which permitted preoperative diagnosis of breast cancer (with postoperative histological confirmation) in all cases, and underwent the same radiotherapy and surgery. Adjuvant chemotherapy (cyclophosphamide methotrexate 5-fluoroacil) was given to patients with unfavourable factors such as axillary lymph node disease, absence of oestrogen receptors, or grade III cytoprognosis (unfavourable prognosis) $^{8}$ or histoprognosis. Most of the remaining patients received adjuvant hormonal treatment, except the patients with grade I cytoprognosis (favourable prognosis). There were no follow up data for two patients, leaving 96 patients.

Imprint slides were made from the resected tumour at the time of surgery, immediately

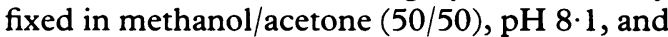
stained according to a hypochromic Papanicolaou staining procedure, ${ }^{10}$ which delicately outlines the distribution of chromatin and 
visualises nucleoli. One imprint from each patient was considered. Nine slides could not be measured because of low cellularity and one slide because of poor quality, so that 86 cases remained.

On each slide an area with the most atypical cells was selected and marked. In these areas vertical zones of $70 \mu \mathrm{m}$ in width were scanned using an ocular grid. All clearly outlined, malignant nuclei whose centre of gravity was within these lines were measured, as well as their nucleoli (defined as conspicuous nuclear bodies with an area $1.0 \mu \mathrm{m}^{2}$ or greater) up to a sample size of 100 cells per slide. A preliminary methodological experiment performed in one of the slides (previously described in detail ${ }^{11}$ ) to assess experimentally the required sample size, showed that with this sample size highly reproducible results (standard error of the mean $<2 \%$ ) for both nuclear and nucleolar area could be obtained. In these 100 nuclei the total number of nucleoli was also registered.

The reproducibility of all measurements and countings was further tested by duplicate measurements of all variables in 10 slides. Correlation coefficients were, in general, high (mean nucleolar area 0.99 , SD 0.80 ; total number of nucleoli/100 nuclei 0.98 ), thus confirming the high reproducibility of the measurements. Poorly stained, or fixed, and clotted nuclei were not measured. All measurements were carried out on a graphic tablet linked to a Mop Videoplan (Kontron, Munich, West Germany) at a final magnifica-

Prognostic significance of the 43 morphometric variables

\begin{tabular}{|c|c|c|c|}
\hline & & p Value & $\begin{array}{l}\text { Mantel Cox } \\
\text { statistic }\end{array}$ \\
\hline \multicolumn{4}{|l|}{ Nuclear variables } \\
\hline Area & $\begin{array}{l}\text { Mean } \\
\text { SD }\end{array}$ & $\begin{array}{l}0.01 \\
\text { NS }\end{array}$ & $\begin{array}{l}9.02 \\
5.86\end{array}$ \\
\hline \multirow[t]{2}{*}{ Perimeter } & Mean & 0.05 & $6 \cdot 22$ \\
\hline & SD & 0.04 & $6 \cdot 32$ \\
\hline \multirow[t]{2}{*}{ Long axis } & Mean & NS & $5 \cdot 55$ \\
\hline & SD & 0.04 & 6.38 \\
\hline \multirow[t]{2}{*}{ Short axis } & Mean & 0.008 & 9.63 \\
\hline & $\mathrm{SD}$ & 0.005 & $10 \cdot 64$ \\
\hline \multirow{2}{*}{ Axis ratio } & Mean & NS & 3.94 \\
\hline & SD & NS & 3.83 \\
\hline \multirow[t]{2}{*}{ Bending energy } & Mean & NS & $5 \cdot 36$ \\
\hline & SD & NS & $4 \cdot 60$ \\
\hline \multirow[t]{2}{*}{ Diameter } & Mean & 0.03 & $7 \cdot 21$ \\
\hline & SD & 0.05 & 6.00 \\
\hline \multirow[t]{2}{*}{ Shape factor } & Mean & NS & 3.09 \\
\hline & $\mathrm{SD}$ & NS & $3 \cdot 81$ \\
\hline \multicolumn{4}{|l|}{ Nucleolar variables: } \\
\hline Area & Mean & 0.05 & $6 \cdot 13$ \\
\hline \multirow{2}{*}{ Perimeter } & Mean & $\begin{array}{l}0.006 \\
0.009\end{array}$ & $\begin{array}{l}7 \cdot 58 \\
9 \cdot 42\end{array}$ \\
\hline & SD & 0.04 & 6.51 \\
\hline \multirow[t]{2}{*}{ Long axis } & Mean & 0.02 & $8 \cdot 33$ \\
\hline & SD & NS & 4.58 \\
\hline \multirow[t]{2}{*}{ Short axis } & Mean & 0.01 & 8.88 \\
\hline & SD & 0.03 & $7 \cdot 35$ \\
\hline \multirow[t]{2}{*}{ Axis ratio } & Mean & NS & $0 \cdot 28$ \\
\hline & SD & NS & 1.79 \\
\hline \multirow[t]{2}{*}{ Bending energy } & Mean & NS & $2 \cdot 47$ \\
\hline & SD & NS & 2.00 \\
\hline \multirow[t]{2}{*}{ Diameter } & Mean & 0.03 & $7 \cdot 30$ \\
\hline & SD & NS & 5.97 \\
\hline \multirow[t]{2}{*}{ Shape factor } & Mean & NS & 1.00 \\
\hline & SD & NS & 0.00 \\
\hline \multicolumn{2}{|l|}{ Nucleolar frequency } & 0.004 & 11.07 \\
\hline \multirow{2}{*}{$\begin{array}{l}\text { Combined variables: } \\
\text { Ratio }\end{array}$} & & & \\
\hline & $\begin{array}{l}\text { Mean } \\
\text { SD }\end{array}$ & $\begin{array}{l}\text { NS } \\
\text { NS }\end{array}$ & $\begin{array}{l}3.05 \\
0.54\end{array}$ \\
\hline \multirow[t]{2}{*}{ Eccentricity } & Mean & NS & $1 \cdot 13$ \\
\hline & SD & NS & 5.07 \\
\hline \multirow{5}{*}{\multicolumn{2}{|c|}{$\begin{array}{l}0 \text { nuclei without nucleoli } \\
0 \text { "nuclei with } 1 \text { nucleolus } \\
0 \text { "nuclei with } 2 \text { nucleoli } \\
0 \text { "nuclei } \\
0 \text { nuclei with } 3 \text { nucleoli } \\
0 \text { " nuclei with }>3 \text { nucleoli } \\
0, \text { nuclei }\end{array}$}} & 0.005 & $10 \cdot 46$ \\
\hline & & NS & 4.87 \\
\hline & & NS & 5.55 \\
\hline & & 0.04 & 6.54 \\
\hline & & 0.004 & $8 \cdot 29$ \\
\hline
\end{tabular}

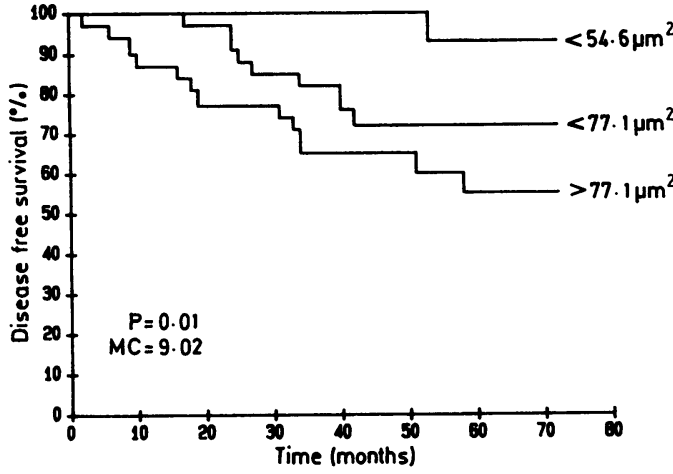

Figure 1 Kaplan-Meier survival curves of patients subdivided according to nuclear area.

tion of 2800 times (1000 times microscope magnification, projection factor of $2 \cdot 8$ ). At this magnification, even nucleoli can be accurately measured. ${ }^{12}$

A total of 42 morphometric variables were considered (table) including nuclear and nucleolar features, nucleolar frequency (expressed as the total number of nucleoli per 100 nuclei), and combined nuclear/nucleolar variables such as nucleolar:nuclear area ratio and nucleolar eccentricity.

For statistical analysis, recurrence free survival (defined as the time between date of operation and local recurrence or the first metastasis) was used as a follow up variable. The follow up term was cut off at six years as very few patients had longer follow up.

Univariate survival analysis was performed according to Kaplan-Meier distribution. For this analysis the patients were divided into three groups of roughly the same size on the basis of measurement values. Differences between the curves were analysed using the Mantel-Cox test, with p values below 0.05 as significant. Multivariate analysis to evaluate the additional prognostic value of the features was performed with the Cox regression model.

All analyses were carried out with the BMDP statistical package, ${ }^{13}$ using the program's life tables $\left(\mathbf{P}_{1} \mathbf{l}\right)$ and survival analysis with covariates $\left(\mathbf{P}_{2} \mathbf{l}\right)$.

\section{Results}

The table shows the results of the univariate Mantel-Cox survival analysis. Eight nuclear variables, nine nucleolar variables, and three

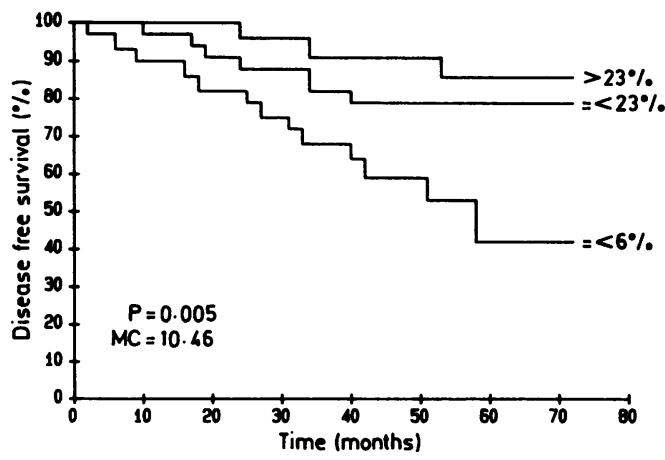

Figure 2 Kaplan-Meier survival curves of patients subdivided according to percentage of nuclei without nucleoli. 
Figure 3 Kaplan-Meier survival curves of patients subdivided according to nucleolar area.

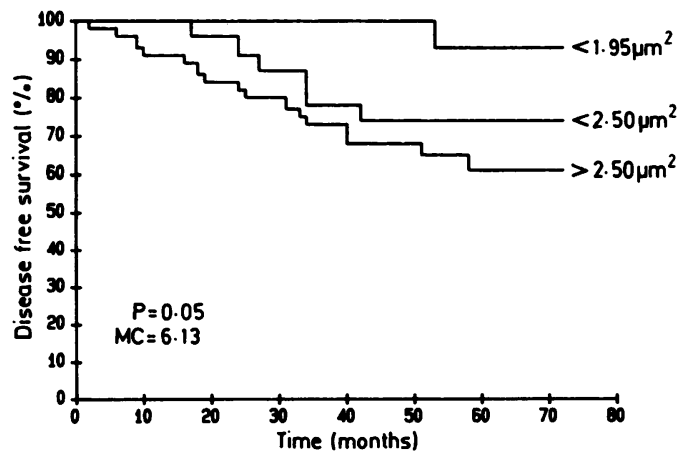

combined variables gave significant $p$ values. Figs 1-4 show the survival curves of the most significant variables.

Multivariate analysis showed that no variable had additional prognostic value once the total number of nucleoli per 100 nuclei was considered.

\section{Discussion}

Of the significant nucleolar variables, nucleolar frequency and the standard deviation of the nucleolar area (SDNA) were the best prognostic variables. The strong prognostic value of the SDNA is in agreement with previous studies on nucleoli in histological sections of breast cancer, ${ }^{9}$ lymphomas, ${ }^{7}$ and ocular melanomas. ${ }^{5}$ The prognostic value of nucleolar frequency was recently observed in prostatic tumours. ${ }^{6}$ The previously reported prognostic value of the SD of nuclear area ${ }^{14}$ could not be reproduced in this study. This may have been due to differences in fixation and staining techniques or in patient selection.

In multivariate analysis only one feature emerged: the total number of nucleoli per 100 nuclei. None of the other variables was selected in multivariate analysis, indicating that the rejected variables contain no additional prognostic information. This variable is the more interesting because its assessment requires no special equipment, and the reproducibility is excellent.

It was very interesting to observe that in this study the nucleolar variables were more powerful from a prognostic point of view than the traditional nuclear variables such as area. Nuclear area is believed to be correlated with differentiation..$^{15} 16$ It is tempting to speculate on which type of malignant biological behaviour the nucleolar features are dependent. Because of the active role of the nucleolus

Figure 4 Kaplan-Meier survival curves of patients subdivided according to total number of nucleoli per 100 nuclei.

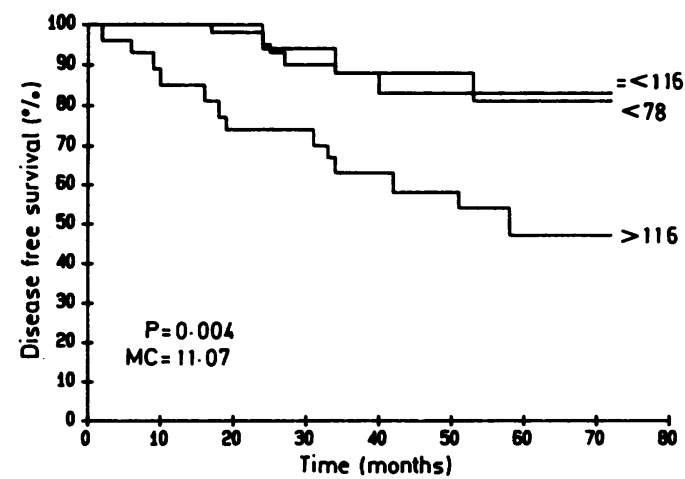

in cell metabolism ${ }^{17}$ nucleolar characteristics may be related to increased cell motility and therefore metastatic potential, or they may be related to high proliferative activity as both biological processes require increased cell metabolism. Previous studies on the morphology of mouse mammary tumour cells in vitro have shown that the increase in size and number of nucleoli is not related to cell multiplication but to cell secretion, such as viral production. ${ }^{18}$ The results of this study therefore suggest that nucleolar frequency may be correlated with metastatic potential. The correlation between nucleolar frequency and number of AgNORs has yet to be clarified.

We conclude that nucleolar morphometric variables assessed in cytological breast cancer specimens give good prognostic information, possibly exceeding that of nuclear variables. Whether these variables offer additional prognostic value to the well established prognostic value of histological morphometric variables and classic variables such as grade, tumour size, and lymph node state should be the subject of further investigation.

We thank Dr S Pearson for help with the manuscript. This study was supported by grant 28-1398 of the Praeventiefonds.

1 Bonadonna G, Rossi A, Valagussa P. Adjuvant CMF chemotherapy in operable breast cancer: ten years later. Lancet 1985;ii:976-7.

2 Baak JPA, Dop H van, Kurver PHJ, Hermans J. The value of morphometry to classic prognosticators in breast cancer. Cancer 1985;56:374-82.

3 Fallenius AG, Auer GU, Carstensen JM. Prognostic significance of DNA measurements in 409 consecutive breast cancer patients. Cancer 1988;62:331-41.

4 Linden JC van der, Lindeman J, Baak JPA, Meijer CJLM, Herman CJ. The multivariate prognostic index and nuclear DNA content are independent prognostic factors in primary breast cancer patients. Cytometry 1989;10: 56-61.

5 Gamel JW, McLean IW. Computerized histopathologic assessment of malignant potential II. A practical method for predicting survival following enucleation for uveal melanoma. Cancer 1983;52:1032-8.

6 Helpap B. Observations on the number, size and localization of nucleoli in hyperplastic and neoplastic prostatic disease. Histopathol 1988;13:203-11.

7 Valk P van der, Mosch A, Kurver PJ, Meijer CJLM. Morphometric characterization of $52 \mathrm{~B}$ cell nonHodgkin's lymphomas. J Clin Pathol 1983;36:289-97.

8 Mouriquand J, Gozlan-Fior M, Villemain D, et al. Value of cytoprognostic classification in breast carcinomas. J Clin Pathol 1986;39:489-96.

9 Baak JPA. The relative prognostic significance of nucleolar morphometry in invasive ductal breast cancer. Histopathol 1985;9:437-44.

10 Mouriquand J, Mouriquand C, Petitpas E, Louis J, Mermet MA. Differential nucleolar staining affinity with a modified Papanicolaou staining procedure. Stain Technol 1981;56:215-9.

11 Diest PJ van, Smeulders AWM, Thunnissen FBJM, Baak JPA. Cytomorphometry: a methodological study on preparation techniques, selection methods and sample size. Analyt Quant Cytol Histol 1989;11:225-31.

12 Fleege JC, Baak JPA, Smeulders AWM. Analysis of measuring system parameters that influence reproducibility of morphometric assessments with a graphic tablet. Hum Pathol 1988;19:513-7.

13 BMDP statistical software manual. Los Angeles: University of California Press, 1985.

14 Kuenen-Bouwmeester V, Hop WCJ, Blonk DI, Boon ME. Prognostic scoring using morphometry and lymph node status of patients with breast carcinoma. Eur J Cancer Clin Oncol 1984;20:337-45.

15 Baak JPA, Persijn JP. In search for the best qualitative microscopical or morphometrical predictor of oestrogen microscopical or morphometrical predictor of oestrogen receptor

16 Diest PJ van, Mouriquand J, Risse EKJ, Schipper NW, Baak JPA. Comparison of lightmicroscopic grading and morphometric features in cytological breast cancer specimens. Pathol Res Pract (in press)

7 Busch H, Smetana K. The nucleolus. New York: Academic Press, 1970.

18 Mouriquand J. De la cellule mammaire. In: Bachet T, Mouriquand J, Favier M, Juvin E, eds. Progrès en pathologie Mammaire. Paris: Formation Médical Continue en Senologie, 1989:52-61. 